\title{
Balloon sinuplasty in one-day surgery
}

\author{
Tatiana Fijałkowska-Ratajczak ${ }^{1}$, Jakub Kopeć ${ }^{1}$, Małgorzata Leszczyńska², Łukasz Borucki ${ }^{1}$ \\ ${ }^{1}$ Department of Otorhinolaryngology, Franciszek Raszeja City Hospital, Poznan, Poland \\ ${ }^{2}$ Department of Otolaryngology and Laryngological Oncology, Poznan University of Medical Sciences, Poznan, Poland
}

Videosurgery Miniinv 2021; 16 (2): 423-428

DOI: https://doi.org/10.5114/wiitm.2021.103962

\begin{abstract}
Introduction: Balloon sinus dilation is becoming an increasingly common procedure, finding a niche as an invaluable tool in endoscopic sinus surgery.

Aim: To assess the effectiveness of balloon sinus dilation (BSD) in 1-day surgery in patients with chronic rhinosinusitis without polyps based on our own experience.

Material and methods: The study group consisted of 9 patients. The Lund-Mackay scale for computed tomography of the paranasal sinuses was assessed prior to surgery. Endoscopy was performed on the basis of the Lund-Kennedy scale and patients were asked to perform the SNOT-22 survey. Two patients underwent balloon sinuplasty under general and seven under local anaesthesia.

Results: Twelve maxillary and eight frontal sinuses were widened (four attempts were unsuccessful). The SNOT-22 survey was assessed the day after surgery (average of 8 points) and a month after surgery (average of 15.5 points). Conclusions: BSD only allows widening of the ostia of the maxillary, frontal and sphenoid sinuses. BSD offers shorter post-surgical recovery, and a more rapid return to work because of its less invasive and less traumatic nature.
\end{abstract}

Key words: one-day surgery, local anaesthesia, chronic rhinosinusitis, balloon sinuplasty, balloon sinus dilation.

\section{Introduction}

Chronic rhinosinusitis (CRS) is a common disease. It is characterised by at least two co-occurring symptoms lasting for at least 12 consecutive weeks, such as the feeling of obstruction, discoloured nasal discharge (anterior/posterior nasal rhinitis), facial pain, and reduction or loss of smell. CRS can be differentiated into two types - with or without polyps - on the basis of endoscopic examination and the presence of polyps in the middle nasal meatus. The pathophysiology of CRS is associated with improper functioning of the sinonasal unit, and the pathology of this region leads to impaired drainage and ventilation of the sinuses [1].

CRS affects $4-28 \%$ of the European and American population. According to EPOS 2012 guidelines, pa- tients presenting with a positive computed tomography (CT) scan, who do not improve after pharmacological treatment, should consider pursuing surgical treatment [1]. The mainstay functional treatment, until recently, consisted of functional endoscopic sinus surgery (FESS) performed under general anaesthesia. However, FESS is considered unsuitable for patients with concomitant diseases, especially those related to the heart, lungs, and central nervous system (most commonly hypertension, arrhythmias, COPD, and bronchial asthma), but also the kidneys, liver, and blood coagulation system. General anaesthesia is also associated with a higher risk to the patient, and it generates higher costs for the hospital due to the requirements for additional staff, specialised equipment and medications, and extended

\section{Address for correspondence}

Tatiana Fijałkowska-Ratajczak, Department of Otorhinolaryngology, Franciszek Raszeja City Hospital, Poznan, Poland,

phone: +48 602526 069, e-mail: tatiana.fijalkowska@gmail.com 
time of hospitalisation. Lastly, FESS poses a burden to the national budget due to the aforementioned factors, as well as increased absence from work due to sickness and hospitalization. An alternative procedure that is available to a select group of patients with chronic rhinosinusitis without polyps (CRSsNP) is balloon sinuplasty or balloon sinus dilation (BSD) surgery, which enables the dilatation of sinus ostia under local anaesthesia [2].

BSD is a technique that allows for the treatment of recurrent acute rhinosinusitis (RARS) and CRSsNP. BSD involves the introduction of a balloon to a given sinus ostium. The balloon is then filled with fluid to a pressure of several atmospheres, which widens the ostia without the need for tissue removal. This is a mechanism that produces microfractures of the bones forming the sinonasal unit while preserving the mucosa $[2,3]$. This facilitates effective sinus irrigation and emptying of sinus contents. Fluoroscopy has been used in the past to confirm correct positioning of the balloon catheter at the sinus ostium, i.e. X-ray radiation from the $\mathrm{C}$ arm. Today, the lighted guidewire system is preferred to illuminate the catheterised sinus (transillumination) [2] followed by insertion of a flexible fibreoptic telescope [4]. Currently, Acclarent [5] or Medtronic [6] navigated balloons are used.

Minimisation of tissue trauma is the main advantage of BSD, and this is particularly important when clearing the frontal recess, because classic FESS often causes scarring and atresia of the ostium when performed here [7]. Patency improvement of this region after balloon sinuplasty also appears to be more permanent [8]. Low tissue traumatisation during surgery equates to less pain. As a result, BSD can be performed on an outpatient basis. However, because complications such as cardiac arrhythmias may arise with the use of local agents, it is standard in our department to perform this procedure under general anaesthesia [9]. Furthermore, conversion to classical FESS should always be considered when performing BSD, especially when encountering anatomical variants such as frontoethmoidal recess, frontal bulla, frontal cell, or Agger nasi cells, as well as patients with suspected oncological changes. With BSD, special attention should be paid to CT scans before surgery [10].

Balloon sinuplasty is not a suitable procedure for all patients with CRS. Furthermore, this method is ineffective for the therapy of ethmoid disease, only allowing for widening of the ostia of maxillary, frontal, and sphenoid sinuses. Current indications for BSD include CRSSNP that does not respond to pharmacological treatment, recurrent acute rhinosinusitis (RARS), CRS in patients with haemorrhagic diathesis, and CRS in patients with frontal sinus obstruction, who are considered high risk for general anaesthesia. This latter group also includes patients in intensive care units with fever as a result of sinus infection [8]. BSD exclusion criteria are as follows: CRS with polyps, mycoses, connective tissue diseases, ethmoidal inflammation, patients with headaches who do not meet the criteria for CRS or RARS, and patients with sinonasal complaints without changes in computed tomography [3].

Balloon sinus dilation is becoming an increasingly common procedure, finding a niche as an invaluable tool in endoscopic sinus surgery. In selected cases, it is an alternative to classic FESS. From 2011 to 2014 in the United States, where traditional endoscopic sinus surgery remained the most frequently performed procedure in the course of CRS, the use of BSD as an independent procedure increased from $5 \%$ to $22.5 \%$ [11].

\section{Aim}

The aim of the study was to assess the effectiveness of balloon sinuplasty in 1-day surgery in patients with chronic rhinosinusitis without polyps, based on our own experience (SNOT analysis-22) [12] and analysis of world and Polish literature using the PubMed database (search criteria: balloon sinus dilation [BSD]).

In the SNOT-22 survey, 22 patients evaluated 22 ailments related to CRS. Out of these 22 ailments, patients selected up to five of the most significant ailments (the need for nasal cleansing, nasal patency, sneezing, runny nose, cough, post-nasal drip, cough with phlegm, feeling of fullness in the ears, dizziness, earache, facial pain/feeling of pressure in the face, impaired sense of smell/taste, difficulty falling asleep, waking up at night, feeling of worse sleep quality, waking up tired, fatigue, decreased productivity, decreased concentration, frustration/ restlessness/irritation, sadness, and embarrassment) and ranked them on a scale of 0 to $5[12,13]$.

\section{Material and methods}

The study group consisted of 9 patients with chronic sinusitis without polyps with craniofacial pain, hospitalised in the Department of Otorhino- 
laryngology of the Raszeja City Hospital in Poznan and qualified for balloon sinuplasty. All procedures were performed between January and August of 2019. All qualified patients agreed to participate in the study and completed the SNOT-22 survey. The study group consisted of 3 men and 6 women aged 27 to 71 years, with an average age of 45.1 years. The study excluded patients under 18 years of age and patients with congenital and acquired immunodeficiencies (congenital neutropenia, autoimmune diseases such as sarcoidosis, lupus erythematosus, psoriasis, arthritis, HIV infection), ciliary dyskinesia, and acute upper and lower respiratory tract infection. Seven patients complained of headache as the predominant symptom, three reported impaired nasal patency, and three reported a feeling of discharge down the back of the throat. One patient had previous FESS and septoplasty, and another patient had previous septoconchoplasty. Three patients had a history of maxillary sinus punctures. Co-morbidities were found in 3 patients: hypertension, multiple sclerosis, and bilateral blindness resulting from congenital atrophy of the optic nerve.

The Lund-Mackay scale for computed tomography of the paranasal sinuses [14] (values range from 2 to 8 points, average 4.6 points) was assessed before surgery. Endoscopy was performed on the basis of the Lund-Kennedy scale [15] (values range from 2 to 8 points, average 4.2 points), and patients were asked to perform the SNOT-22 survey (the range of values was 23 to 63 points, average 37.33 points).

The Lund-Mackay scale estimates the severity of changes in computed tomography of the paranasal sinuses. All paranasal sinuses are divided into right and left, and assessed with regards to the presence of: polyps (on a scale of 0 to 2 points, where 0 is no polyps, 1 is polyps limited to the middle nasal meatus, and 2 indicates polyps in the nasal cavity), oedema (where 0 is no oedema, 1 is mild oedema, and 2 is oedematous hypertrophy), and secretions (where 0 is no secretions, 1 is clear secretions, and 2 is thick and/or mucopurulent discharge) [14].

The Lund-Kennedy score assesses the extent of changes in computed tomography of the paranasal sinuses. All paranasal sinuses are divided into right and left, and are assessed in terms of: sinus shading (on a scale of 0 to 2 , where 0 is a sinus without deviations, 1 partial, and 2 total shadowing) and patency of the ostiomeatal complex (on a scale of 0 to 2 , where 0 is patent and 2 is occluded) [15].
Patients underwent balloon sinuplasty under local and general anaesthesia: 2 patients under general anaesthesia and seven under local anaesthesia. In 1 case, the decision to perform general anaesthesia was dictated by the necessity to remove anterior ethmoid sinus osteomas, and in the second case because the patient presented in our department for the first time.

Local anaesthesia was performed according to the protocol used in our ward for other rhinological procedures. Forty-five minutes prior to surgery, local anaesthesia was administered to both nasal passages: Betadrin (1 ml contains: $1 \mathrm{mg}$ of diphenhydramine hydrochloride, $0.33 \mathrm{mg}$ of naphazoline nitrate) with $2 \%$ lignocaine in a $50 / 50$ ratio. In addition, patients received the following premedication: Dormicum 11.25 mg p.o. and Ketonal (ketoprofen) $100 \mathrm{mg}$ intravenously or metamizole $2.5 \mathrm{~g}$ intravenously. In the operating room, $2 \%$ lignocaine packing with adrenaline at a concentration of $1: 1000$ was inserted for 2 min into the central nasal cavities and along the lower nasal turbinate (six drops - about $0.1 \mathrm{ml}$ of adrenaline per $20 \mathrm{ml}$ of $2 \%$ lignocaine).

Injections were made at points that are typical for FESS - $20 \mathrm{ml}$ of $1 \%$ lignocaine with four drops of adrenaline (concentration of adrenaline in $1: 100,000$ lignocaine solution) at:

- the region of the palatine-ganglionic ganglia,

- the "armpit" of the middle nasal turbinate,

- along the hook process,

- the head of the middle nasal turbinate,

- the front, middle, and back of the lower nasal turbinate.

The next step was blockade of the second branch of the trigeminal nerve - anaesthesia through the larger palatal opening - location as indicated in Photo 1. First, submucosal anaesthesia with $1 \%$ lignocaine with the addition of adrenaline at a concentration of $1: 100,000$ (approx. $1 \mathrm{ml}$ ) was administered, followed by the introduction of the anaesthetic agent directly into the canal in the amount of about $8 \mathrm{ml}$.

The last step was blockade of the first branch of the trigeminal nerve - injection in the region of the supraorbital opening, using 2-4 $\mathrm{ml}$ of $1 \%$ lidocaine with the addition of $1: 100,000$ adrenaline (Photo 2).

The procedure lasted between 15 and $50 \mathrm{~min}$. Rigid 0 - and 30-degree endoscopes and navigated balloons (Medtronic NuVent EM Balloon Sinus Dilation System BSDS) were used. Under CT navigation 


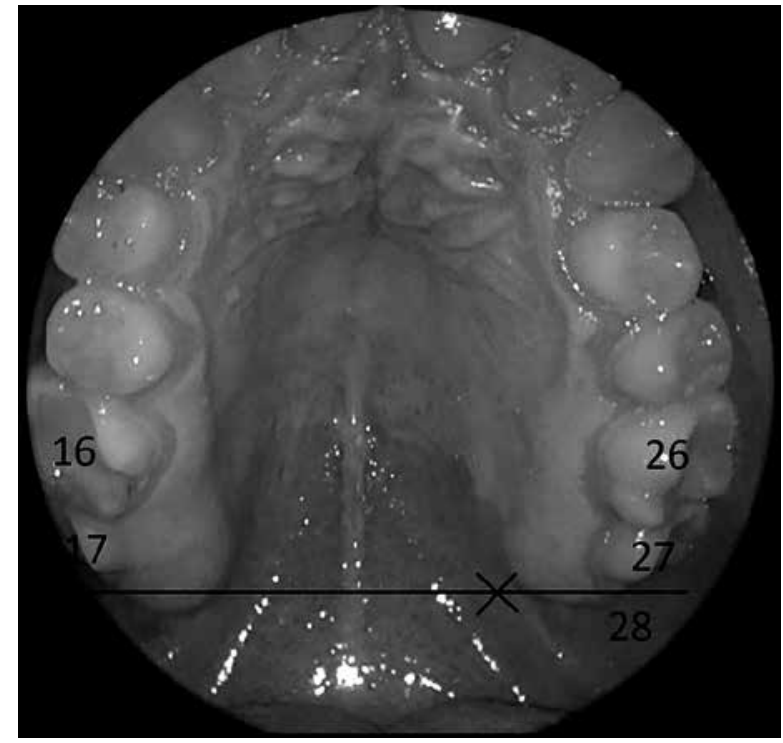

Photo 1. Location of the larger palatal opening

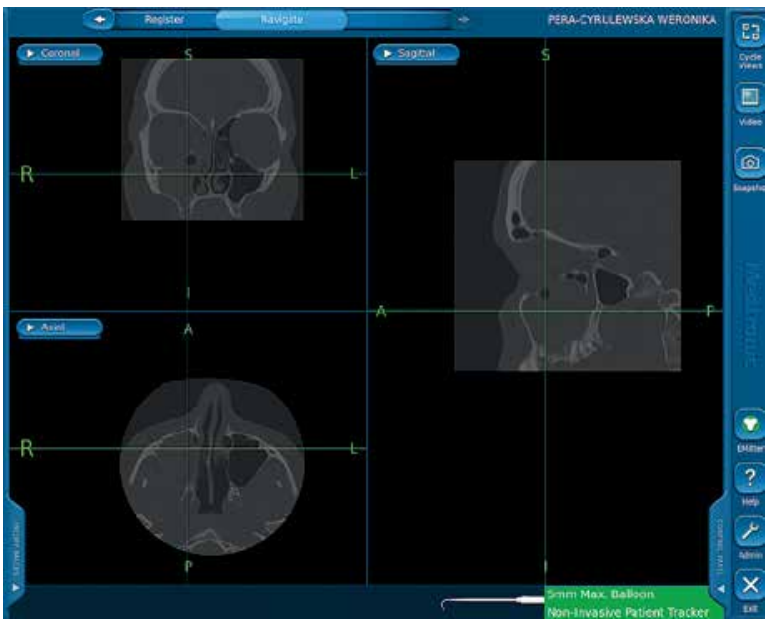

Photo 3. Computed tomography navigation control of BSDS

control, BSDS was introduced to the ostia of the maxillary or frontal sinus. The position was verified by navigation, and the BSDS was expanded with water, reaching a pressure of $10 \mathrm{~atm}$ for $10 \mathrm{~s}$. After the procedure, endoscopic control was performed (Photos 3 and 4).

\section{Results}

In total, 20 paranasal sinuses were widened (average 2.22 per patient) - 12 maxillary sinuses - eight left, five right, and four opened on both sides. The frontal ostia were widened eight times - the frontal

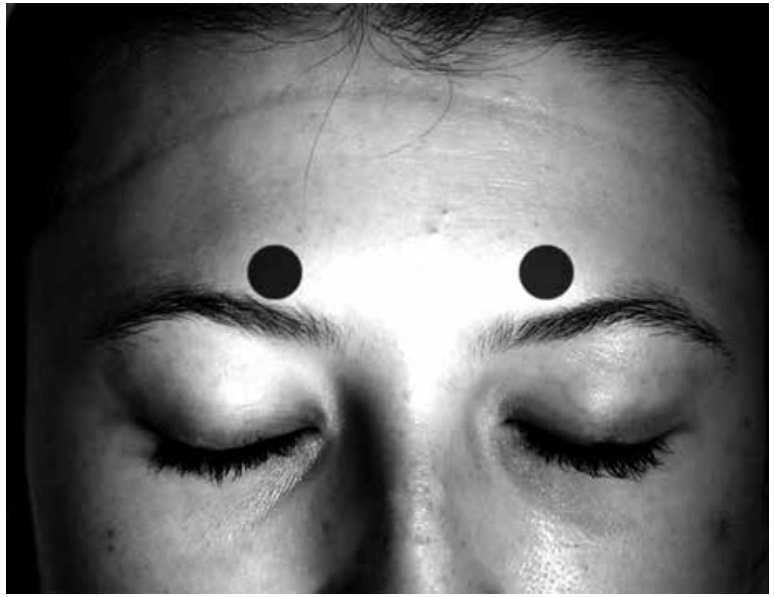

Photo 2. Location of the supraorbital opening

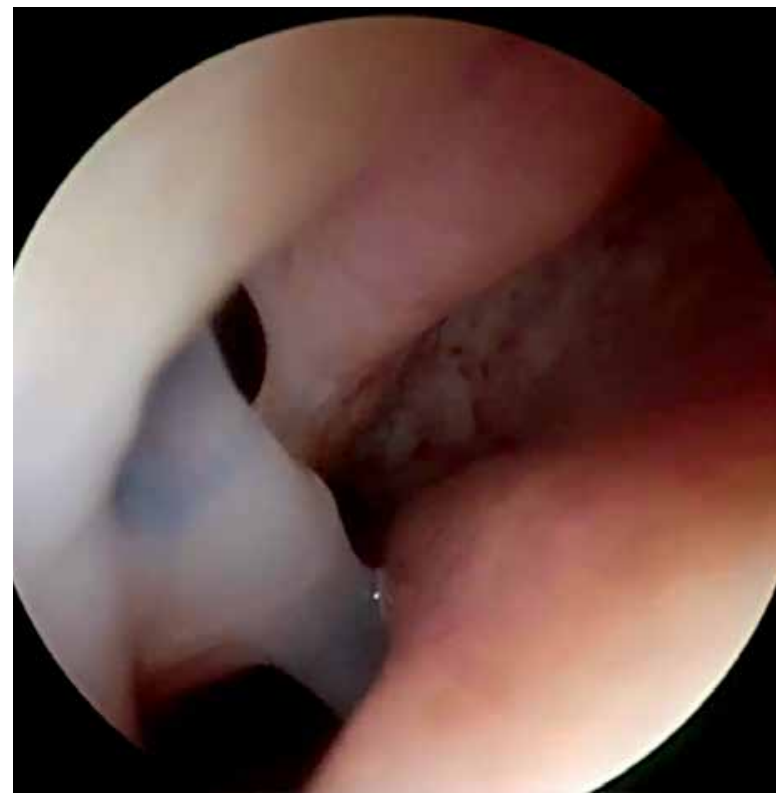

Photo 4. Endoscopic control after the BSDS

sinus was opened four times on both sides, and four attempts were unsuccessful. Two patients underwent additional procedures. In 1 patient, the left medial nasal turbinate was medialised with the hook process lifted to remove an osteoma from the recess, and in the other patient, polyps were removed from the left anterior recess.

The SNOT-22 survey was assessed the day after surgery and ranged from 2 to 41 points (average 8 points). Three patients were not evaluated. SNOT-22 evaluated a month after surgery ranged from 6 to 41 points (average of 15.5 points). Three patients were again not evaluated. 


\section{Discussion}

Currently, solutions are being sought that allow for effective, and at the same time the least invasive treatment of known diseases - from chronic paranasal sinusitis or mucoceles of the maxillary sinuses or choanal polyps, to rare cases such as nasal dermoid sinus cysts.

In concurrence with the patients who underwent balloon sinuplasty at our department, the improvement of patient ailments has been well established by many larger studies. It also seems that the robustness of treatment effects, for which there is currently insufficient evidence, is good. Our study group had a statistically significant improvement in SNOT-22. Before surgery it averaged 37.33, 1 month after surgery it was 8 , and 3 months after surgery it was 15.5. Koskinen et al. published a report about the persistence of effects and patient satisfaction 7 years after surgery. In these studies, the less invasive and less traumatic nature of balloon sinuplasty (balloon sinus dilation BSD) is emphasised in direct comparison to the FESS procedure [16-18].

Despite the high cost of the necessary equipment, BSD offers shorter post-surgical recovery and a faster return to work as economic justification. It can be successfully performed as a procedure requiring a 1-day hospital stay. Bowles et al. were able to discharge patients from the ward on the day of surgery, despite the use of general anaesthesia [19]. In Finland, cocaine is the local anaesthetic agent used, but it is not available in Poland. The protocol for local anaesthesia developed in our department produced satisfactory results and patient comfort, regardless of the ostium requiring dilatation, while allowing patient discharge from the department on the same day. Further statistical evaluation of this anaesthetic technique is currently being performed.

\section{Conclusions}

Headache was the predominant symptom in the majority of respondents (seven out of nine). Six patients improved after brief discomfort. Marzetti et al. published work involving a larger number of patients, and similarly demonstrated the effectiveness of balloon sinuplasty in the treatment of headaches associated with sinusitis or nasal inflammation [20, 21]. This may be due to the re-establishment of physiological sinus airflow and more effective pressure equalisation [22], suggesting that these ailments are among the basic indications for balloon sinuplasty. Nevertheless, it should be noted that while balloon sinuplasty of the maxillary ostia is not difficult, the catheterisation of the frontal ostia, in contrast, is characterised by greater difficulty and a higher failure rate. In our study, $50 \%$ of attempts were unsuccessful; the literature reports this percentage in the range of $6-19 \%$ [10], due to high anatomic variability [23]. The frontal ostia are also sensitive to mucosal damage, which in turn may exacerbate ostial obstruction and increase the risk of re-stenosis (29\% 6 years after surgery) [10]. Therefore, it is important to thoroughly evaluate CT imaging, in which the discrepancy in interpretation between the isolated frontal projection and all three CT planes can be as high as $40 \%$ [23], as well as to establish appropriate qualification of patients. The much more extensive Draf I procedure (endoscopic frontal recess approach) remains another option with many potential complications. In our study there were no postoperative complications; the study by Cingia et al. suggests a complication rate of $0.01 \%$ [3]. Balloon sinuplasty also seems to be a good alternative to FESS for patients who meet the treatment criteria, due to the smaller number of adhesions [24].

\section{Conflict of interest}

The authors declare no conflict of interest.

\section{References}

1. Fokkens WJ, Lund VJ, Mullol J, et al. European position paper on rhinosinusitis and nasal polyps 2012. Rhinol Suppl 2012; 23 : 1-298.

2. Dariusz J, Szczygielski K, Brzozowski K. Cewnikowanie endoskopowe zatok - ewolucja czy rewolucja w leczeniu zapaleń zatok przynosowych? Doświadczenia własne i przegląd literatury. Otolaryngol Pol 2009; 63: 113-7.

3. Cingia C, Bayar Mulukb N, Lee JT. Current indications for balIoon sinuplasty. Curr Opin Otolaryngol Head Neck Surg 2019; 27: 7-13.

4. Lam, K, Bigcas JL, Luong A, et al. Flexible microsensor technology for real-time navigation tracking in balloon sinus ostial dilation. Allergy Rhinol 2017; 8: 20-4.

5. https://www.acclarent.com/solutions/products/trudi-navigation-system/relieva-spinplus-NAV.

6. https://www.medtronic.com/us-en/healthcare-professionals/products/ear-nose-throat/balloon-sinus-dilation/nuvent-em-balloon-sinus-dilation-system.html.

7. Eloy JA, Friedel ME, Eloy JD, et al. In-office balloon dilation of the failed frontal sinusotomy. Otolaryngol Head Neck Surg 2012; 146: 320-2. 
8. Szczygielski K, Galusza B, Rapiejko P, et al. A six-month analysis of frontal sinus drainage pathway in patients with frontal sinusitis after balloon sinuplasty. Acta Otolaryngologica 2017; 137: 968-74.

9. Hughes N, Bewick J, Van Der Most R, O’Connell M. A previous ly unreported serious adverse event during balloon sinuplasty. BMJ Case Rep 2013; 2013: bcr2012007879.

10. Heimgartner S, Eckardt J, Simmen D, et al. Limitations of balloon sinuplasty in frontal sinus surgery. Eur Arch Otorhinolaryngol 2011; 268: 1463-7.

11. Chaaban MR, Baillargeon JG, Baillargeon G, et al. Use of balloon sinuplasty in patients with chronic rhinosinusitis in the United States. Int Forum Allergy Rhinol 2017; 7: 600-8.

12. Hopkins C, Browne JP, Slack R, et al. The national comparative audit of surgery for nasal polyposis and chronic rhinosinusitis. Clin Otolaryngol Allied Sci 2006; 31: 390-9.

13. https://www.canvasc.ca/pdf/SNOT22.pdf.

14. Lund VJ, Mackay IS. Staging in rhinosinusitis. Rhinology 1993; 107: $183-4$

15. Lund VJ, Kennedy DW. Quantification for staging sinusitis. The Staging and Therapy Group. Ann Otol Rhinol Laryngol Suppl 1995; 167: 17-21.

16. Koskinen A, Myller J, Mattila P, et al. Long-term follow-up after ESS and balloon sinoplasty: comparison of symptom reduction and patient satisfaction. Acta Otolaryngol 2016; 136: 532-6.

17. Waniewska-Leczycka M, Cieslik T, Popko M. Combined minimally invasive surgical management of a nasal dermoid sinus cyst affecting the frontal sinus: literature review and new classification. Videosurgery Miniinv 2020; 15: 632-44.

18. Koskinen A, Myller J, Mattila P, et al. Long-term follow-up after ESS and balloon sinoplasty: comparison of symptom reduction and patient satisfaction. Acta Otolaryngol 2016; 136: 532-6.

19. Bowles P, Agrawal S, Salam M. Efficacy of balloon sinuplasty in treatment of frontal rhinosinusitis: a prospective study in sixty patients. Clin Otolaryngol 2017; 42: 908-11.

20. Marzetti A, Mazzone S, Tedaldi M, et al. The role of balloon sinuplasty in the treatment of vacuum rhinogenic headache. Indian J Otolaryngol Head Neck Surg 2017; 69: 216-20.

21. Marzetti A, Tedaldi M, Passali F. The role of balloon sinuplasty in the treatment of sinus headache. Otolaryngol Pol 2014; 68: 15-9.

22. Bizaki AJ, Numminen J, Taulu R, Rautiainen M. Decrease of nasal airway resistance and alleviations of symptoms after balloon sinuplasty in patients with isolated chronic rhinosinusitis: a prospective, randomised clinical study. Clin Otolaryngol 2016; 41: $673-80$

23. Koskinena A, Mattilab P, Myller J, et al. Comparison of intra-operative characteristics and early post-operative outcomes between endoscopic sinus surgery and balloon sinuplasty. Acta Otolaryngol 2017; 137: 202-6.

24. Leunig A, Betz CS, Sommer B, Sommer F. Anatomic variations of the sinuses; multiplanar CT-analysis in 641 patients. Laryngorhinooologie 2008; 87: 482-9.

Received: 25.03.2020, accepted: 1.11.2020. 